\title{
CUTTING MARKOVIAN TREES
}

\author{
Paavo Salminen
}

Åbo Akademi, Matematiska Institutionen, SF-20500 Åbo, Finland

\begin{abstract}
In this paper a path transformation of a branching Markov process is studied in which, roughly speaking, all finite branches are erased with unit speed from the tip of the branch for a given time $\varrho, 0 \leq \varrho \leq \infty$. To have a more applicable touch we, in fact, transform killed branching processes. Killing is done at a terminal line. This notion is introduced as a generalization of the notion of terminal time. It is proved that the transformed process is again a branching Markov process and its law is characterized. This may be viewed as a generalization of a classical result of Harris [5], and a more recent result of Neveu [9].
\end{abstract}

\section{Introduction}

To start with we describe shortly a result of Harris [5] (see also Athreya and Ney $[2 ;$ p. 50$])$. Let $Z:=\left\{Z_{t}: t \geq 0\right\}$ be a Galton-Watson process with the offspring distribution $\left\{p_{k}: k=0,2, \ldots\right\}$. Let $F$ be the generating function of $p$. Then the probability of extinction, $q$, is the smallest nonnegative root of the equation $t=F(t)$. Assume $0<q<1$, and introduce

$$
\hat{F}(s):=\frac{F((1-q) s+q)-q}{1-q}, \quad 0 \leq s \leq 1 .
$$

It is easily seen that $\hat{F}$ is the generating function of the distribution $\hat{p}$ with

$$
\begin{aligned}
& \hat{p}_{k}=\frac{1}{1-q} \sum_{l \geq k}\left(\begin{array}{l}
l \\
k
\end{array}\right)(1-q)^{k} q^{l-k} p_{l}, \quad k=1,2, \ldots, \\
& \hat{p}_{0}=0 .
\end{aligned}
$$

Let $\hat{Z}$ be a Galton-Watson process with the offspring distribution $\hat{p}$. Because $\hat{p}_{0}=0$ the probability of extinction for $\hat{Z}$ is zero.

To describe a path transformation which leads to $\hat{Z}$ we introduce some terminology adapted from Neveu [8].

Definition 1. A tree $\omega$ is a subset of the space

$$
U:=\bigcup_{n=1}^{\infty} \mathbf{N}_{+}^{n} \cup\{\emptyset\}, \quad \mathbf{N}_{+}:=\{1,2, \ldots\},
$$

satisfying the conditions

(1) $\emptyset \in \omega$,

(2) if $u v \in \omega$ then $u \in \omega$,

(3) for all $u \in \omega$ there exists $\nu^{u}(\omega) \in \mathbf{N}_{+}$such that if $u j \in \omega$ for some $j \in \mathbf{N}_{+}$ then $1 \leq j \leq \nu^{u}(\omega)$. 
Elements in $U$ are called particles. To explain the notation $u v$ in (2), let $u=\left(i_{1}, \ldots, i_{s}\right) \in U, v=\left(j_{1}, \cdots, j_{r}\right) \in U$ then $u v=\left(i_{1}, \ldots, i_{s}, j_{1}, \ldots, j_{r}\right) \in U$. The variable $\nu^{u}$ in (3) gives the number of descendants of the particle $u$. A particle $v$ is called an ancestor of a particle $u$, denoted $v \leq u$, if there exists $w \in U$ such that $u=v w$. Defining $\emptyset u=u$ and $u \emptyset=u$ it is seen that for every $u \in U$ we have $u \leq u$ and $\emptyset \leq u$.

Every particle $u$ in a tree $\omega$ is now marked with a positive number $\zeta^{u}$, called the lifetime of $u$. Further, the life interval of $u$ is defined to be $\left(\alpha^{u}, \beta^{u}\right)$, where

$$
\begin{aligned}
\alpha^{u} & :=\sum_{v<u} \zeta^{v}, \quad \alpha^{\emptyset}:=0, \\
\beta^{u} & :=\sum_{v \leq u} \zeta^{v}=\alpha^{u}+\zeta^{u} .
\end{aligned}
$$

The space of all marked trees is denoted with $\Omega$, and is used as the canonical sample space for nonexplosive Galton-Watson processes. Elements in $\Omega$ are still denoted with $\omega$, and we write $u \in \omega$ meaning that $u$ is a particle in the marked tree $\omega$.

For $u \in U$ and $\omega \in \Omega$ let $\Omega^{u}:=\{\omega: u \in \omega\}$, and introduce a mapping $\theta^{u}: \Omega^{u} \rightarrow \Omega$ by setting $v \in \theta^{u} \circ \omega$ if and only if $u v \in \omega$. We say that the branch starting from $u$ in $\omega$ is finite if $\omega^{u}:=\theta^{u} \circ \omega$ is a finite tree, i.e. the number of particles in $\omega^{u}$ is finite. The space of all finite marked trees is denoted with $\Omega_{f}$.

Define a mapping $e: \Omega \rightarrow \Omega \cup\{\dagger\}$, where $\dagger$ stands for the void tree, as follows:

(i) In the case $\omega \notin \Omega_{f}$ let $u \in e \circ \omega$ if and only if $u \in \omega$ and $\omega^{u} \notin \Omega_{f}$.

(ii) In the case $\omega \in \Omega_{f}$ let $e \circ \omega=\dagger$.

In other words, $e \circ \omega$ contains only the infinite branches of the tree $\omega$.

Let $\mathbf{P}_{p, \alpha}$ be the law of a nonexplosive Galton-Watson process $Z$. Here $p$ denotes the offspring distribution and $\alpha$ is the parameter for the exponentially distributed lifetimes. We are interested in the image of the law $\mathbf{P}_{p, \alpha}$ under the mapping $e$. The following result is extracted from Athreya and Ney [2; p. 49].

Theorem 1. For $A \in \mathscr{W}:=\sigma\left\{\nu^{u}, \zeta^{u}: u \in U, \omega \in \Omega^{u}\right\} \cup\{\dagger\}$

$$
\hat{\mathbf{P}}_{p, \alpha}(\omega \in A):=\mathbf{P}_{p, \alpha}(e \circ \omega \in A)=q \varepsilon_{\{\dagger\}}(A)+(1-q) \mathbf{P}_{\hat{p}, \alpha}(A),
$$

where the offspring distribution $\hat{p}$ is given in (1.1) and $\varepsilon_{\{\dagger\}}$ is Dirac's measure at $\dagger$.

More recently, Neveu [9] considered a transformation, called erasure, operating on the space of finite trees. To recall this let $\zeta(\omega):=\sup _{u \in \omega} \beta^{u}$ be the lifetime of a tree $\omega$, and for $0<\varrho<\infty$ define a mapping $e_{\varrho}: \Omega_{f} \rightarrow \Omega_{f} \cup\{\dagger\}$ as follows:

(i) $u \in e_{\varrho} \circ \omega$ if and only if $u \in \omega$ and $\zeta\left(\theta^{u} \circ \omega\right) \geq \varrho$.

(ii) For all $u \in e_{\varrho} \circ \omega$ set $\zeta^{u}\left(e_{\varrho} \circ \omega\right)=\min \left\{\zeta^{u}(\omega), \zeta\left(\theta^{u} \circ \omega\right)-\varrho\right\}$.

(iii) $e_{\varrho} \circ \omega=\dagger$, if $\zeta(\omega)<\varrho$.

For (sub)critical Galton-Watson processes we have (see Neveu [9]) 
Theorem 2. For $A \in \mathscr{W}:=\sigma\left\{\nu^{u}, \zeta^{u}: u \in U, \omega \in \Omega^{u}\right\} \cup\{\dagger\}$

$$
\hat{\mathbf{P}}_{p, \alpha}^{\varrho}(\omega \in A):=\mathbf{P}_{p, \alpha}\left(e_{\varrho} \circ \omega \in A\right)=\lambda_{\varrho} \varepsilon_{\{\dagger\}}(A)+\left(1-\lambda_{\varrho}\right) \mathbf{P}_{\hat{p}, \alpha}^{\varrho}(A),
$$

where $\lambda_{\varrho}:=\mathbf{P}_{p, \alpha}(\zeta<\varrho)$ and the measure $\mathbf{P}_{\hat{p}, \alpha}^{\varrho}$ governs a Galton-Watson process having the offspring distribution $\hat{p}^{\varrho}$ given by

$$
\hat{p}_{k}^{\varrho}:=\frac{1}{1-\lambda_{\varrho}}\left\{\sum_{l=k}^{\infty} p_{l}\left(\begin{array}{l}
l \\
k
\end{array}\right) \lambda_{\varrho}^{l-k}\left(1-\lambda_{\varrho}\right)^{k}-\lambda_{\varrho} \varepsilon_{\{0\}}(k)\right\}, \quad k=0,1, \ldots
$$

It is quite obvious that Theorem 2 is also valid for nonexplosive supercritical Galton-Watson processes, there is no problem to extend $e_{\varrho}$ to $\Omega$. Note, however, that infinite branches are not cut. Further, because $q=\lim _{\varrho \rightarrow \infty} \lambda_{\varrho}$, Theorem 1 is obtained in some sense from the extended version of Theorem 2.

The aim of this note is to generalize Neveu's result to a larger class of branching Markov processes. In Ikeda, Nagasawa and Watanabe [7; Section 5.5] one finds a generalization of Harris' transformation, see also Hering [6] or Asmussen and Hering $[1 ;$ p. 242] . In these works the approach is analytic and the point of view of a path transformation is not adopted.

In the next section we introduce the notion of terminal line. A terminal line is "the initial point" for the erasure. In the third section erasure or cutting theorems are proved. This is done under some regularity assumptions which allow us, among other things, to use the Martin-boundary theory. In [10] these results are applied to branching Brownian motion processes to obtain a ratio limit theorem for the number of particles in the erased process and the original one. This generalizes a result in Athreya and Ney [2; p. 51].

\section{Terminal lines}

Let $\Omega$ be the space of marked trees as defined above. The particles $u \in U$ are now additionally marked with "cadlag" functions $\gamma^{u}: I^{u} \rightarrow E$, where $E$ is a locally compact space having a countable base and $I^{u}:=\left[0, \zeta^{u}\right), \omega \in \Omega^{u}$. Further, we set $\gamma_{0}^{u j}=\gamma_{\zeta-}^{u}$ for $1 \leq j \leq v^{u}$ and $\gamma_{t}^{u}=\Delta$ for all $t \geq \zeta^{u}$ or $\gamma_{t}^{u}=\partial$ for all $t \geq \zeta^{u}$ where $\Delta$ and $\partial$ are fictitious states isolated from $E$. This new space is still denoted with $\Omega$ and is used as the canonical path space for a non-explosive branching Markov process $X$. We assume that the process $X$ has a constant creation rate $\alpha$, i.e. for every $u \in U$ the lifetime $\zeta^{u}$ is exponentially distributed with parameter $\alpha$. Particles are sent to the state $\Delta$ at creation times. For this process there is no use for the extra cemetery point $\partial$. The non-branching part, $\mathbf{x}$, of $X$ is taken to be an exponentially with parameter $\alpha$ killed conservative standard process having the state space $E \cup\{\Delta\}$. In the next section further regularity assumptions are imposed. The process $\mathbf{x}$ is considered in the canonical sample space $(\mathbf{D}, \mathscr{D})$ of cadlag functions, denoted $\gamma$, having the cemetery points $\Delta$ 
and $\partial$ as above. Finally, the offspring distribution, $\pi(y):=\left\{p_{n}(y), n=0,2, \ldots\right\}$, $y \in E$, of $X$ is assumed to be such that $X$ is non-explosive (for a condition see Athreya and Ney [2; p. 104]).

Next we introduce some relevant $\sigma$-algebras on $\Omega$ (cf. Chauvin [3]). Firstly, for every $u \in U$ let

$$
\mathscr{F}_{t}^{u}:=\sigma\left\{\gamma_{s}^{u}(\omega): 0 \leq s<t \wedge \zeta^{u}(\omega), \omega \in \Omega^{u}\right\},
$$

and define recursively

$$
\begin{aligned}
& \mathscr{G}^{\emptyset}:=\sigma\left\{\gamma_{0}^{\emptyset}: \omega \in \Omega^{\emptyset}=\Omega\right\}, \\
& \mathscr{G}^{u}:=\mathscr{G}^{v} \vee \mathscr{F}_{\infty}^{v} \vee \sigma\left\{\nu^{v}: \omega \in \Omega^{v}\right\},
\end{aligned}
$$

where $v$ is $u$ 's parent, i.e. there exists $j \in \mathbf{N}$ such that $u=v j$. Intuitively, $\mathscr{G}^{u}$ contains the information on the branch leading to the particle $u$. To include the history of the particle itself set

$$
\begin{aligned}
& \mathscr{A}_{s}^{u}:=\mathscr{G}^{u} \vee \mathscr{F}_{s}^{u}, \\
& \mathscr{A}_{\infty}^{u}:=\vee_{s>0} \mathscr{A}_{s}^{u} \vee \sigma\left\{\nu^{u}: \omega \in \Omega^{u}\right\} .
\end{aligned}
$$

The following notions are introduced in Chauvin [3].

Definition 2. A family $\tau:=\left\{\tau^{u}: u \in U\right\}, \tau^{u}:\left(\Omega^{u}, \mathscr{A}_{\infty}^{u}\right) \rightarrow\left[0, \zeta^{u}\right] \cup\{\infty\}$, of non-negative random variables is called a stopping line if

(i) for all $u \in U \quad \tau^{u}$ is a stopping time for $\mathscr{A}^{u}:=\left\{\mathscr{A}_{s}^{u}: s \geq 0\right\}$,

(ii) given a particle $u \in L_{\tau}(\omega):=\left\{u: \tau^{u}(\omega)<\infty\right\}$, $\omega \in \Omega$, there does not exist a particle $v$ such that $v<u$ and $v \in L_{\tau}(\omega)$.

Remark. This definition differs slightly from the one in [3] in that $\tau^{u}$ may attain "the value" $+\infty$, and, therefore, $u \in L_{\tau}$ in the case $\tau^{u}=\zeta^{u}$.

Definition 3. A mapping $\theta_{t}^{u}: \Omega^{u} \cap\left\{\zeta^{u}>t\right\} \rightarrow \Omega, u \in U, t \geq 0$, is called a shift operator if it has the properties:

(i) $v \in \theta_{t}^{u} \circ \omega$ if and only if $u v \in \omega$.

(ii) If $u v \in \omega, v \neq \emptyset$, and $\zeta^{u}>t$ then $\gamma^{v}\left(\theta_{t}^{u} \circ \omega\right)=\gamma^{u v}(\omega)$.

(iii) $\zeta^{\emptyset}\left(\theta_{t}^{u} \circ \omega\right)=\zeta^{u}(\omega)-t$.

(iv) If $0 \leq s<\zeta^{u}-t$ then $\gamma_{s}^{\emptyset}\left(\theta_{t}^{u} \circ \omega\right)=\gamma_{s+t}^{u}(\omega)$.

Let $\tau$ be a stopping line. Then we introduce random shift operators $\theta_{\tau}^{u}: \Omega^{u} \cap$ $\left\{\zeta^{u}>\tau^{u}\right\} \rightarrow \Omega, u \in U$, as "deterministic" shift operators above having $\tau^{u}$ in the place of $t$.

For the next definition let for $\omega \in \Omega$ and a stopping line $\tau$

$$
D_{\tau}(\omega):=\left\{u \in U: \text { there exists } v \in U, v<u, v \in L_{\tau}(\omega)\right\},
$$

where $\tau$ is a stopping line. The set $D_{\tau}$ is called the set of strict descendents of the line $\tau$. 
Definition 4. A stopping line $\tau$ is called a terminal line if $u \notin D_{\tau}(\omega)$ and $t<\tau^{u}(\omega)$ implies that $t+\tau^{\emptyset} \circ \theta_{t}^{u}=\tau^{u}$, where $\omega \in \Omega^{u}$ and $0 \leq t<\zeta^{u}$.

Definition 5. Let $\tau$ be a terminal line. A mapping $\kappa_{\tau}: \Omega \rightarrow \Omega$ is called a killing operator if it has the properties

(i) $v \in \kappa_{\tau} \circ \omega$ if and only if $v \in \omega, v \notin D_{\tau}(\omega)$,

(ii) for $v \in \kappa_{\tau} \circ \omega$

if $v \notin L_{\tau}(\omega)$ then $\gamma^{v}\left(\kappa_{\tau} \circ \omega\right)=\gamma^{v}(\omega)$,

if $v \in L_{\tau}(\omega)$ then

$$
\gamma_{t}^{v}\left(\kappa_{\tau} \circ \omega\right)= \begin{cases}\gamma_{t}^{v}(\omega), & t<\tau^{v} \\ \partial, & t \geq \tau^{v}\end{cases}
$$

Using the definitions above it is easily seen that we have the following, intuitively obvious but fundamental, result.

Proposition 1. Let $\tau$ be a terminal line. Then $\theta_{s}^{u} \circ \kappa_{\tau} \circ \omega=\kappa_{\tau} \circ \theta_{s}^{u} \circ \omega$, where $u \in \kappa_{\tau} \circ \omega$ and $0 \leq s<\tau^{u} \wedge \zeta^{u}$.

Let $\mathscr{F}:=\left\{\mathscr{F}_{t}: t \geq 0\right\}$ be the canonical filtration in $\Omega$ satisfying the usual conditions and generated basically by the variables

(i) $\left\{\nu^{u}, \zeta^{u}, \gamma^{u}\right\}: \Omega^{u} \rightarrow\left(\mathbf{Z}_{+}, \operatorname{Re}_{+}, \mathbf{D}\right)$ for $\alpha^{u}+\zeta^{u} \leq t$,

(ii) $\left\{\gamma_{s}^{u}: \alpha^{u}+s \leq t<\alpha^{u}+\zeta^{u}\right\}$.

For a terminal line $\tau$ we also introduce the "stopped" $\sigma$-algebras $\mathscr{G}:=\left\{\mathscr{G}_{t}\right.$ : $t \geq 0\}$ satisfying the usual conditions and generated by the variables

(i) $\left\{\nu^{u}, \zeta^{u}, \gamma^{u}\right\}: \Omega^{u} \rightarrow\left(\mathbf{Z}_{+}, \operatorname{Re}_{+}, \mathbf{D}\right)$ for $\alpha^{u}+\zeta^{u} \leq t$ and $u \notin D_{\tau} \cup L_{\tau}$,

(ii) $\left\{\gamma_{s}^{u}: \alpha^{u}+s \leq t \wedge\left(\alpha^{u}+\tau^{u}\right), u \in L_{\tau}\right\}$.

Denote with $\mathbf{P}$. the measure on $\left(\Omega, \mathscr{F}_{\infty}\right)\left(\mathscr{F}_{\infty}:=\vee_{t \geq 0} \mathscr{F}_{t}\right)$ induced by the process $X$. Let $\tau$ be a terminal line. We are interested in the image of $\mathbf{P}$. under the mapping $\kappa_{\tau}$, denoted with $\hat{\mathbf{P}}$., i.e. for $A \in \mathscr{F}_{\infty}$

$$
\hat{\mathbf{P}} .(\omega \in A)=\mathbf{P} .\left(\kappa_{\tau} \circ \omega \in A\right) .
$$

Let $\hat{\tau}(\gamma):=\tau^{\emptyset}(\omega)$, where the particle $\emptyset$ in the tree $\omega$ carries the function $\gamma$ as the mark. Then, $\hat{\tau}$ may be and is considered as a terminal time for the process $\mathbf{x}$. The measure induced by $\mathbf{x}$ on $(\mathbf{D}, \mathscr{D})\left(\mathscr{D}:=\vee_{t \geq 0} \mathscr{D}_{t}, \mathscr{D}_{t}:=\sigma\left\{\gamma_{s}: s \leq t\right\}\right.$ with the usual conditions) is denoted with $\mathbf{P}^{\mathbf{x}}$. Let $\hat{\mathbf{P}}^{\mathbf{x}}$. be the law of the process $\hat{\mathbf{x}}$ obtained from $\mathbf{x}$ by killing it at $\hat{\tau}$, i.e. the sample paths of $\hat{\mathbf{x}}$ are

$$
\hat{\gamma}_{t}= \begin{cases}\gamma_{t}, & t<\zeta \wedge \hat{\tau} \\ \Delta, & \zeta \leq t, \quad \zeta \leq \hat{\tau}, \\ \partial, & \hat{\tau} \leq t, \hat{\tau}<\zeta .\end{cases}
$$

We assume that 
(A) the process $\left(\hat{\mathbf{x}}, \hat{\mathbf{P}}^{\mathbf{x}}\right)$ is a standard Markov process on the state space $\left(\hat{E}_{\tau}, \mathscr{E}_{\tau}\right)$, where $\hat{E}_{\tau}:=E_{\tau} \cup\{\Delta, \partial\}, E_{\tau}:=\left\{x \in E: \mathbf{P}_{x}^{\mathbf{x}}(\hat{\tau}=0)=0\right\}$ and $\mathscr{E}_{\tau}$ is the associated $\sigma$-algebra on $\hat{E}_{\tau}$.

See Sharpe [11; p. 71] for the conditions for this to hold. Clearly, (A) is a property for the terminal time $\hat{\tau}$ and, hence, also for the terminal line $\tau$.

Proposition 2. Under (A) the measure $\hat{\mathbf{P}}$. governs on the space $\left(\Omega, \mathscr{G}_{\infty}\right)$ a non-explosive branching Markov process, denoted $\hat{X}$. Its non-branching part is identical in law with $\hat{\mathbf{x}}$, and the offspring distributions of $\hat{X}$ and $X$ coincide, i.e. for $k=0,2,3, \ldots$

$$
\hat{\mathbf{P}} .\left(\nu^{\emptyset}=k \mid \gamma_{\zeta}^{\emptyset}=\Delta\right)=\mathbf{P} .\left(\nu^{\emptyset}=k \mid \gamma_{\zeta}^{\emptyset}=\Delta\right) .
$$

Proof. The claim concerning the offspring distribution is obviously true. Note, however, that $\hat{\mathbf{P}} .\left(\nu^{\emptyset}=0\right) \neq \mathbf{P} .\left(\nu^{\emptyset}=0\right)$. To verify the Markov property let for $t>0 L_{t}:=\left\{u: \alpha^{u} \leq t<\beta^{u}\right\}$ be the population living at time $t$. Then defining

$$
\sigma^{u, t}:= \begin{cases}t-\alpha^{u}, & \text { for } \alpha^{u} \leq t<\beta^{u} \\ \infty, & \text { for } t<\alpha^{u} \text { or } \beta^{u} \leq t\end{cases}
$$

it is seen that $\sigma^{t}:=\left\{\sigma^{u, t}: u \in U\right\}$ is a stopping line and $L_{\sigma^{t}} \equiv L_{t}$ (cf. [3]). Further, set $\tilde{\theta}_{t}^{u}:=\theta_{\sigma^{t}}^{u}$ and $\tilde{\gamma}_{t}^{u}:=\gamma_{\sigma^{t}}^{u}$. Let now $G$, and for every $u \in U$, $F^{u}$ be positive, bounded $\mathscr{G}_{t}$-and, respectively, $\mathscr{G}_{\infty}$-measurable random variables. Consider, for $x \in E_{\tau}$,

$$
\begin{aligned}
\hat{\mathbf{E}}_{x}\left(G \Pi_{u \in L_{t}} F^{u} \circ \tilde{\theta}_{t}^{u}\right)=\mathbf{E}_{x}\left(G \circ \kappa_{\tau} \Pi_{u \in L_{t} \circ \kappa_{\tau}} F^{u} \circ \tilde{\theta}_{t}^{u} \circ \kappa_{\tau}\right) \\
=\mathbf{E}_{x}\left(G \circ \kappa_{\tau} \Pi_{u \in L_{t} \circ \kappa_{\tau}} F^{u} \circ \kappa_{\tau} \circ \tilde{\theta}_{t}^{u}\right) \\
=\mathbf{E}_{x}\left(G \circ \kappa_{\tau} \mathbf{E}_{x}\left(\Pi_{u \in L_{t} \circ \kappa_{\tau}} F^{u} \circ \kappa_{\tau} \circ \tilde{\theta}_{t}^{u} \mid \tilde{\gamma}_{t}^{u} \circ \kappa_{\tau}, u \in L_{t} \circ \kappa_{\tau}\right)\right) \\
=\hat{\mathbf{E}}_{x}\left(G \hat{\mathbf{E}}_{x}\left(\Pi_{u \in L_{t}} F^{u} \circ \tilde{\theta}_{t}^{u} \mid \tilde{\gamma}_{t}^{u}, u \in L_{t}\right)\right),
\end{aligned}
$$

where we have used Proposition 1 and the Markov property of $X$. This proves the Markov property of $\hat{X}$. For the branching property we have

$$
\begin{aligned}
\hat{\mathbf{E}}_{x}\left(\Pi_{u \in L_{t}} F^{u} \circ \tilde{\theta}_{t}^{u} \mid \mathscr{G}_{t}\right) & =\hat{\mathbf{E}}_{x}\left(\Pi_{u \in L_{t}} F^{u} \circ \tilde{\theta}_{t}^{u} \mid \tilde{\gamma}_{t}^{u}, u \in L_{t}\right) \\
& =\mathbf{E}_{x}\left(\Pi_{u \in L_{t}} F^{u} \circ \kappa_{\tau} \circ \tilde{\theta}_{t}^{u} \mid \tilde{\gamma}_{t}^{u} \circ \kappa_{\tau}, u \in L_{t} \circ \kappa_{\tau}\right) \\
& =\Pi_{u \in L_{t} \circ \kappa_{\tau}} \hat{\mathbf{E}}_{\hat{X}_{t}^{u}}\left(F^{u}\right),
\end{aligned}
$$

where we have used the Markov property of $\hat{X}$, Proposition 1 , and the branching property of $X$. Finally, it is easily seen that the non-branching part of $\hat{X}$ is as claimed. Thus, the proof is complete.

Remark. This result is intuitively obvious and certainly well known. The proof above is given to show how the introduced machinery works, in this respect. 


\section{Cutting a tree}

1. Let $\tau$ be a terminal line and $\kappa_{\tau}$ the killing operator associated with it. Further, let

$A=\left\{\omega:\right.$ for every $v \in \kappa_{\tau} \circ \omega$ there exists $u$ such that $\left.v \leq u, u \in L_{\tau}\right\}$.

For a killed tree $\kappa_{\tau} \circ \omega$ we define now the extinction time $T$ via

$$
T(\omega)= \begin{cases}\sup \left\{\alpha^{u}+\tau^{u}: u \in L_{\tau}(\omega)\right\}, & \text { if } \omega \in A, \\ \infty, & \text { otherwise }\end{cases}
$$

As an example consider the following family of random variables

$$
\tau^{u}:= \begin{cases}\zeta^{u}, & \text { if } \nu^{u}=0 \\ \infty, & \text { otherwise }\end{cases}
$$

Clearly, this is a terminal line and the corresponding extinction time is the "usual" one.

Definition 6. For a given $\varrho \geq 0$ a mapping $c_{\varrho}: \Omega \rightarrow \Omega \cup\{\dagger\}$ is called a cutting or erasure operator if it has the properties

(a) $v \in \omega^{\varrho}:=c_{\varrho} \circ \kappa_{\tau} \circ \omega$ if and only if $v \in \kappa_{\tau} \circ \omega, T\left(\theta_{0}^{v} \circ \kappa_{\tau} \circ \omega\right)>\varrho$,

(b) for $v \in \omega^{\varrho}$ :

(i) $\zeta^{v}\left(\omega^{\varrho}\right)=\min \left\{\zeta^{v}\left(\kappa_{\tau} \circ \omega\right), T\left(\theta_{0}^{v} \circ \kappa_{\tau} \circ \omega\right)-\varrho\right\}$,

(ii) $\gamma_{t}^{v}\left(\omega^{\varrho}\right)= \begin{cases}\gamma_{t}^{v}(\omega), & \text { if } 0 \leq t<\zeta^{v}\left(\omega^{\varrho}\right) ; \\ \partial, & \text { if } \zeta^{v}\left(\omega^{\varrho}\right) \leq t \text { and } \zeta^{v}\left(\omega^{\varrho}\right)=T\left(\theta_{0}^{v} \circ \kappa_{\tau} \circ \omega\right)-\varrho ; \\ \Delta, & \text { if } \zeta^{v}\left(\omega^{\varrho}\right) \leq t \text { and } \zeta^{v}\left(\omega^{\varrho}\right)=\zeta^{v}\left(\kappa_{\tau} \circ \omega\right) ;\end{cases}$

(c) for $T\left(\kappa_{\tau} \circ \omega\right)<\varrho: c_{\varrho} \circ \kappa_{\tau} \circ \omega=\dagger$.

In other words, informally speaking, in a $c_{\varrho}$-transformed tree $\omega^{\varrho}$ all finite branches are made $\varrho$ units (measured in time from the terminal line) shorter than in the killed tree $\kappa_{\tau} \circ \omega$. Note that the definition of $c_{\varrho}$ makes, after some obvious changes, perfectly good sense also in the case " $\varrho=+\infty$ ". This mapping in which all finite branches (measured from $\tau$ ) are cutted is denoted with $c_{\infty}$.

2. Consider now, as introduced in Section 2, a branching process $\hat{X}=$ $\left\{\hat{X}_{t}, \hat{\mathbf{P}}\right.$. $\}$ killed at a terminal line $\tau$, and recall the notation $\hat{\mathbf{x}}=\left\{\gamma_{t}, \hat{\mathbf{P}}^{\mathbf{x}}\right\}$ for its non-branching part. It is assumed that

(B) the semigroup associated with the process $\hat{\mathbf{x}}$ is a Feller-Dynkin semigroup (in the terminology of Williams [12; p. 115]) having the strong Feller property, i.e. for all $t \geq 0$ and $f$ bounded and measurable the mapping $x \rightarrow \hat{\mathbf{E}}_{x}^{\mathbf{x}}\left(f\left(\gamma_{t}\right)\right)$ $\left(x \in E_{\tau}\right)$ is continuous. 
Moreover, we make use of the Martin-boundary theory to analyse the process $\hat{\mathbf{x}}$ and, therefore, impose the following assumptions to get results strong enough:

(C) (i) $\hat{\mathbf{x}}$ has a jointly measurable transition density with respect to a $\sigma$-finite measure $m$. This density is denoted with $\hat{p}(t ; x, y), t \geq 0, x, y \in E_{\tau}$.

(ii) Green's function

$$
\hat{g}_{\alpha}(x, y):=\int_{0}^{\infty} \exp (-\alpha t) \hat{p}(t ; x, y) d t
$$

exists for every $\alpha \geq 0, x, y \in E_{\tau}$, and is such that the function

$$
\hat{g}_{\alpha}(f, y):=\int_{E_{\tau}} m(d x) f(x) \hat{g}_{\alpha}(x, y)
$$

is continuous and bounded for all $f \in C_{c}\left(E_{\tau}\right)$.

(D) (i) There exists a finite measure $\mu$ on $\mathscr{E}_{\tau}$ such that the function

$$
q(y):=\int_{E_{\tau}} \mu(d x) \hat{g}_{0}(x, y), \quad y \in E_{\tau}
$$

is positive and continuous.

(ii) For every $\alpha \geq 0$ and $f \in C_{c}\left(E_{\tau}\right)$ there exists a constant $c_{f}^{\alpha}$ such that for each $\alpha$-excessive and $\mu$-integrable function $h$ one has

$$
m(f h) \leq c_{f}^{\alpha} \mu(h) .
$$

Under the assumptions (C) and (D) (cf. Dynkin [4]) the state space $E_{\tau}$ can be Martin-compactified in such a way that: Any $\mu$-integrable excessive function $h$ has an integral representation in terms of the minimal excessive functions. Given such a function $h$ the representing measure, denoted with $v^{h}$ is connected with the exit distribution of the $h$-transform of $\hat{\mathbf{x}}$ via the formula

$$
\hat{\mathbf{P}}_{x}^{\mathbf{x}, h}\left(\zeta \in d t, \gamma_{\zeta-} \in A\right)=d t \int_{A} \frac{\hat{p}(t ; x, y)}{h(x)} v^{h}(d z), \quad A \in \mathscr{E}_{\tau}
$$

where $\hat{\mathbf{P}}^{\mathbf{x}, h}$ is the measure associated with the $h$-transform and $\zeta$ is the lifetime of the process. Further, when restricted to $\mathscr{E}_{\tau}, v^{h}$ is absolutely continuous w.r.t. $m$ and satisfies

$$
v^{h}(\cdot)=\lim _{t \downarrow 0} \int \frac{h(y)-\hat{\mathbf{E}}_{y}^{\mathbf{x}}\left(h\left(\gamma_{t}\right)\right)}{t} m(d y)
$$

see $[4 ;$ p. 132].

For simplicity, we consider below the cutting operator in two separate cases:

(3.4a) $\tau^{\emptyset}<\zeta^{\emptyset}$ a.s. on $\left\{\tau^{\emptyset}<\infty\right\}$,

(3.4b) $\tau^{\emptyset}=\zeta^{\emptyset}$ a.s. on $\left\{\tau^{\emptyset}<\infty\right\}$.

The general case is a combination of these. 
3. Assume that the terminal line $\tau$ satisfies the condition (3.4a) above. Here we need the following regularity assumption on the distribution of the extinction time $T$ :

(E) For every $0 \leq \varrho<\infty$ and $x \in E$

$$
\lim _{h \downarrow 0} \frac{1}{h} \mathbf{P}_{x}(\varrho<T \leq \varrho+h)=: a(x, \varrho)
$$

exists and is a locally bounded function of $x$.

Recall the notation $\hat{\tau}$ for the terminal time of the non-branching part $\mathbf{x}$ associated with the terminal line $\tau$ and that $E_{\tau}:=\left\{x \in E: \mathbf{P}_{x}^{\mathbf{x}}(\hat{\tau}=0)=0\right\}$.

Proposition 3. Let $X$ be a branching Markov process and $\tau$ a terminal line as described above. Assume that $X$ (and $\tau$ ) satisfy (A)-(E). Then the function $u^{\varrho}:=\mathbf{P} .(T \geq \varrho)$ is, for every $\varrho \geq 0$ excessive for $\hat{\mathbf{x}}$. Denote the $u^{\varrho}$-transform of $\hat{\mathbf{x}}$ with $\hat{\mathbf{x}}^{\varrho}=\left\{\gamma_{t}, \hat{\mathbf{P}}^{\mathbf{x}, \varrho}\right\}$. Then $\hat{\mathbf{P}}^{\mathbf{x}}, \varrho(\hat{\tau}<\infty)=0$ for $x \notin\{u \varrho=0\}$, and the representing measure $v^{u}$ of the function $u^{\varrho}$ (with the normalization (3.2)) is concentrated on $\mathscr{E}_{\tau}$, i.e. $\hat{\mathbf{P}}^{\mathbf{x}}, \varrho\left(\gamma_{\zeta-} \in E_{\tau}\right)=1$, and given by

$$
v^{u}(\cdot)=\int\left[a(y, \varrho)+\alpha\left(p_{0}(y)+1-F\left(1-u^{\varrho}(y)\right)\right)\right] m(d y),
$$

where $F$ is the generating function of the offspring distribution. have

Proof. We prove first that $u^{\varrho}$ is excessive for $\hat{\mathbf{x}}$. For $t>0,0 \leq \varrho<\infty$ we

$$
\begin{aligned}
u^{\varrho}(x): & =\mathbf{P}_{x}(T \geq \varrho) \geq \mathbf{P}_{x}(T>\varrho+t) \\
& \geq \mathbf{P}_{x}\left(T>\varrho+t, \zeta^{\emptyset}>t, \tau^{\emptyset}>t\right) \\
& =\hat{\mathbf{E}}_{x}^{\mathbf{x}}\left(\mathbf{P}_{\gamma_{t}}(T>\varrho)\right)=\hat{\mathbf{E}}_{x}^{\mathbf{x}}\left(u^{\varrho}\left(\gamma_{t}\right)\right) .
\end{aligned}
$$

Using the assumption (B) it is seen that $x \rightarrow u^{\varrho}(x)$ is continuous. Consequently, by the right continuity of $\hat{\mathbf{x}}$ at 0 and the normality, we obtain

$$
u^{\varrho}(x)=\lim _{t \downarrow 0} \hat{\mathbf{P}}_{x}^{\mathbf{x}}\left(u^{\varrho}\left(\gamma_{t}\right)\right)
$$

Combining (3.6) and (3.7) it is seen that $u^{\varrho}$ is excessive for $\hat{\mathbf{x}}$. Next we prove that the measure $v^{u}$ is concentrated on $\mathscr{E}_{\tau}$ i.e.

$$
\hat{\mathbf{P}}_{x}^{\mathbf{x}, \varrho}\left(\gamma_{\zeta-} \in E_{\tau}\right)=1
$$

For this consider the function $v^{\varrho}:=\hat{\mathbf{P}}_{x}^{\mathbf{x}}(\hat{\tau} \geq \varrho)$. It is easily seen that $v^{\varrho}$ is excessive for $\hat{\mathbf{x}}$. Moreover,

$$
\left\{v^{\varrho}=0\right\} \subseteq\left\{u^{\varrho}=0\right\}
$$


We are interested in the $v^{\ell}$-transform of $\hat{\mathbf{x}}$. To have a probabilistic interpretation of this introduce a measurable mapping $\hat{c}_{\varrho}: \mathbf{D} \rightarrow \mathbf{D} \cup\{\hat{\dagger}\}$, where $\hat{\dagger}$ is the void function, by setting (cf. Definition 6)

(3.10) (a) $\hat{c}_{\varrho}(\gamma)=\gamma$ if $\gamma \in\{\zeta<\hat{\tau}\}$,

(b) $\left(\hat{c}_{\varrho}(\gamma)\right)_{t}=\left\{\begin{array}{ll}\gamma_{t}, & \text { for } t+\varrho<\hat{\tau} ; \\ \partial, & \text { for } t+\varrho \geq \hat{\tau},\end{array}\right.$ if $\gamma \in\{\zeta>\hat{\tau}, \hat{\tau}>\varrho\}$,

(c) $\hat{c}_{\varrho}(\gamma)=\hat{\dagger}$ if $\gamma \in\{\zeta<\hat{\tau}, \hat{\tau} \leq \varrho\}$.

Standard computations show that for $A \in \mathscr{D}$

$$
\hat{\mathbf{P}}_{x}^{\mathbf{x}}\left(\hat{c}_{\boldsymbol{Q}}(\gamma) \in A \mid \hat{c}_{\boldsymbol{e}}(\gamma) \neq \hat{\dagger}\right)=\hat{\mathbf{P}}_{x}^{\mathbf{x}, \boldsymbol{v}}(\gamma \in A)
$$

where $\hat{\mathbf{P}}_{\boldsymbol{x}}^{\mathbf{x}, v}$ denotes the measure of the $v^{\ell}$-transform of $\hat{\mathbf{x}}$. By the definition of the mapping $\hat{c}_{\varrho}$, especially $(3.10 \mathrm{~b})$, and $(3.11)$ we have

$$
\hat{\mathbf{P}}_{x}^{\mathbf{x}, v}\left(\gamma_{\zeta-} \in E_{\tau}\right)=1
$$

By (3.9) the function

$$
h^{\varrho}:=\frac{u^{\varrho}}{v^{\varrho}}
$$

is well-defined on $\left\{u^{\varrho} \neq 0\right\}$. Further, $h^{\varrho}$ is excessive for the $v^{\varrho}$-transform, and the $h^{\mathcal{e}}$-transform of the $v^{\boldsymbol{e}}$-transform is identical in law with the $u^{\boldsymbol{e}}$-transform of $\hat{\mathbf{x}}$. Consequently, the measure $\hat{\mathbf{P}}_{x}^{\mathbf{x}, e}$ is absolutely continuous with respect to the measure $\hat{\mathbf{P}}_{x}^{\mathbf{x}, v}$. Therefore $\hat{\mathbf{P}}_{x}^{\mathbf{x}, v}\left(\gamma_{\zeta} \notin \notin E_{\tau}\right)=0$ implies $\hat{\mathbf{P}}_{x}^{\mathbf{x}, \varrho}\left(\gamma_{\zeta} \notin \notin E_{\tau}\right)=0$, i.e. (3.10), which also gives the claim $\hat{\mathbf{P}}_{x}^{\mathbf{x}, \boldsymbol{e}}(\hat{\tau}<\infty)=0$. To compute the measure $v^{u}$ we use the formula (3.3). Firstly, consider

$$
1-u^{\varrho}(x)=\mathbf{P}_{x}(T \leq \varrho)=\mathbf{P}_{x}\left(T \leq \varrho, \zeta^{\emptyset}>t\right)+\mathbf{P}_{x}\left(T \leq \varrho, \zeta^{\emptyset}<t\right) .
$$

Assume $t<\varrho$, and denote the first term on the right hand side of (3.12) with $T_{1}$ and the second with $T_{2}$. Then

$$
\begin{aligned}
T_{1} & =\mathbf{P}_{x}\left(T \leq \varrho, \zeta^{\emptyset}>t, \tau^{\emptyset}<t\right)+\mathbf{P}_{x}\left(T \leq \varrho, \zeta^{\emptyset}>t, \tau^{\emptyset}>t\right) \\
& =\mathbf{P}_{x}^{\mathbf{x}}(\hat{\tau}<t<\zeta)+\mathbf{P}_{x}^{\mathbf{x}}\left(\hat{\tau} \wedge \zeta>t ; \mathbf{P}_{\gamma_{t}}(T<\varrho-t)\right), \\
T_{2}= & \mathbf{P}_{x}\left(T \leq \varrho, \zeta^{\emptyset}<t, \tau^{\emptyset}<t\right)+\mathbf{P}_{x}\left(T \leq \varrho, \zeta^{\emptyset}<t, \tau^{\emptyset}>t\right) \\
= & \mathbf{P}_{x}^{\mathbf{x}}(\hat{\tau}<\zeta<t) \\
& +\int_{0}^{t} \int_{E} \mathbf{P}_{x}^{\mathbf{x}}\left(\zeta \in d s, \gamma_{\zeta-} \in d y, \hat{\tau}>s\right) \sum_{k=2}^{\infty} p_{k}(y)\left(\mathbf{P}_{y}(T<\varrho-s)\right)^{k},
\end{aligned}
$$


where we have used the assumption $\tau^{\emptyset} \neq \zeta^{\emptyset}$ a.s. on $\left\{\tau^{\emptyset}<\infty\right\}$ and the definition of terminal line $\tau$ in that

$$
\begin{aligned}
& \left\{\zeta^{\emptyset}<t, \tau^{\emptyset}<t\right) \equiv\left\{\tau^{\emptyset}<\zeta^{\emptyset}<t\right\}, \\
& \left\{\zeta^{\emptyset}<t, \tau^{\emptyset}>t\right) \equiv\left\{\zeta^{\emptyset}<t, \tau^{\emptyset}=\infty\right\} \equiv\left\{\zeta^{\emptyset}<t, \zeta^{\emptyset}<\tau^{\emptyset}\right\} .
\end{aligned}
$$

From (3.12) and (3.13) it follows that

$$
\begin{aligned}
u^{\varrho}(y) & -\hat{\mathbf{E}}_{y}^{\mathbf{x}}\left(\mathbf{P}_{\gamma_{h}}(T \geq \varrho-h)\right) \\
& =\int_{0}^{h} \int_{E_{\tau}} \mathbf{P}_{y}^{\mathbf{x}}\left(\zeta \in d s, \gamma_{\zeta-} \in d z, \hat{\tau}>s\right)\left(p_{0}(z)+1-F\left(\mathbf{P}_{z}(T<\varrho-s)\right)\right) .
\end{aligned}
$$

Setting " $\varrho=\varrho+h "$ gives

$$
\begin{aligned}
& u^{\varrho}(y)-\hat{\mathbf{E}}_{y}^{\mathbf{x}}\left(u^{\varrho}\left(\gamma_{h}\right) ; \zeta \vee \hat{\tau}>h\right) \\
& =\mathbf{P}_{y}(\varrho+h \geq T>\varrho)+\int_{0}^{h} \int_{E_{\tau}} \mathbf{P}_{y}^{\mathbf{x}}\left(\zeta \in d s, \gamma_{\zeta-} \in d z, \hat{\tau}>s\right) \\
& \quad \times\left(p_{0}(z)+1-F\left(\mathbf{P}_{z}(T<\varrho+h-s)\right)\right) .
\end{aligned}
$$

Using the assumption (E) and the strong continuity of the Feller-Dynkin semigroup it is seen that

$$
\lim _{h \downarrow 0} \frac{u^{\varrho}(y)-\hat{\mathbf{E}}_{y}^{\mathbf{x}}\left(u^{\varrho}\left(\gamma_{h}\right)\right)}{h}=a(y, \varrho)+\alpha\left(p_{0}(y)+1-F\left(1-u^{\varrho}(y)\right)\right) .
$$

It follows, by the assumption (E), that the dominated convergence theorem is applicable in the formula (3.3) for compact $A \in \mathscr{E}_{\tau}$ with $m(A)<\infty$, and this gives (3.5).

Theorem 3. Let the assumptions in Proposition 2 be valid. Then the image of the measure $\hat{\mathbf{P}}$. under the mapping $c_{\varrho}$ is given by

$$
\hat{\mathbf{P}}_{x}^{\varrho}(\omega \in F)=\hat{\mathbf{P}}_{x}\left(c_{\varrho} \circ \omega \in F\right)=\left(1-u^{\varrho}(x)\right) \varepsilon_{\{\dagger\}}(F)+u^{\varrho}(x) \tilde{\mathbf{P}}_{x}^{\varrho}(\omega \in F),
$$

where $F \in \mathscr{F}_{\infty}$ and the measure $\tilde{\mathbf{P}} \varrho$ governs a branching Markov process having (a) the $u^{\varrho}$-transform of $\hat{\mathbf{x}}$ as the non-branching part,

(b) the offspring distribution:

for $k=1,2, \ldots$

$$
\begin{aligned}
\tilde{\mathbf{P}}^{\varrho}\left(\nu^{\emptyset}\right. & \left.=k \mid \gamma_{\zeta-}^{\emptyset}=y, \gamma_{\zeta}^{\emptyset}=\Delta\right) \\
& =\frac{1}{p_{0}(y)+1-F\left(1-u^{\varrho}(y)\right)} \sum_{l=k}^{\infty} p_{l}(y)\left(\begin{array}{l}
l \\
k
\end{array}\right) u^{\varrho}(y)^{k}\left(1-u^{\varrho}(y)\right)^{l-k},
\end{aligned}
$$

for $k=0$ 


$$
\begin{aligned}
\tilde{\mathbf{P}} \varrho\left(\nu^{\emptyset}\right. & \left.=0 \mid \gamma_{\zeta-}^{\emptyset}=y, \gamma_{\zeta}^{\emptyset}=\Delta\right)=\frac{p_{0}(y)}{p_{0}(y)+1-F(1-u \varrho(y))}, \\
\tilde{\mathbf{P}^{\varrho}} \cdot\left(\gamma_{\zeta}^{\emptyset}\right. & \left.=\Delta \mid \gamma_{\zeta-}^{\emptyset}=y\right)=1-\tilde{\mathbf{P}}^{\varrho} \cdot\left(\gamma_{\zeta}^{\emptyset}=\partial \mid \gamma_{\zeta-}^{\emptyset}=y\right) \\
& =\frac{p_{0}(y)+1-F\left(1-u^{\varrho}(y)\right)}{(a(y, \varrho) / \alpha)+p_{0}(y)+1-F\left(1-u^{\varrho}(y)\right)} .
\end{aligned}
$$

Proof. Consider first the non-branching part. For $0<s_{1}<\cdots<s_{n}$ and $A_{i} \in \mathscr{E}_{\tau}$ we have

$$
\begin{aligned}
& \tilde{\mathbf{P}}_{x}^{\varrho}\left(\gamma_{s_{1}}^{\emptyset} \in A_{1}, \ldots, \gamma_{s_{n}}^{\emptyset} \in A_{n}, s_{n}<\zeta^{\emptyset}\right) \\
&=\hat{\mathbf{P}}_{x}^{\varrho}\left(\gamma_{s_{1}}^{\emptyset} \in A_{1}, \ldots, \gamma_{s_{n}}^{\emptyset} \in A_{n}, s_{n}<\zeta^{\emptyset} \mid \omega \neq \dagger\right) \\
&=\hat{\mathbf{P}}_{x}\left(\gamma_{s_{1}}^{\emptyset} \in A_{1}, \ldots, \gamma_{s_{n}}^{\emptyset} \in A_{n}, s_{n}<\zeta^{\emptyset}, s_{n}<T-\varrho \mid T>\varrho\right) \\
&=\frac{1}{u \varrho(x)} \hat{\mathbf{P}}_{x}\left(\gamma_{s_{1}}^{\emptyset} \in A_{1}, \ldots, \gamma_{s_{n}}^{\emptyset} \in A_{n}, s_{n}<\zeta^{\emptyset}, s_{n}<T-\zeta, T>\zeta\right) \\
&=\frac{1}{u^{\varrho}(x)} \mathbf{P}_{x}^{\mathbf{x}}\left(\gamma_{s_{1}} \in A_{1}, \ldots, \gamma_{s_{n}} \in A_{n}, s_{n}<\zeta \wedge \hat{\tau} ; u^{\varrho}\left(\gamma_{s_{n}}\right)\right),
\end{aligned}
$$

which proves the claim. Next we study branching (cf. Neveu [9]). For $k \geq 1$ let $F_{j} \in \mathscr{F}_{\infty}, j=1, \ldots, k$, and consider

$$
\begin{gathered}
\tilde{\mathbf{P}}_{x}^{\varrho}\left(\nu^{\emptyset}=k, \gamma_{\zeta-}^{\emptyset} \in d y, \zeta^{\emptyset} \in d s, \theta_{0}^{j} \circ \omega \in F_{j}, j=1, \ldots, k\right) \\
=\frac{1}{u \varrho(x)} \mathbf{P}_{x}\left(\nu^{\emptyset} \circ c_{\varrho} \circ \kappa_{\tau}=k, \gamma_{\zeta-}^{\emptyset} \circ c_{\varrho} \circ \kappa_{\tau} \in d y, \zeta^{\emptyset} \circ c_{\varrho} \circ \kappa_{\tau} \in d s,\right. \\
\left.\quad \theta_{0}^{j} \circ c_{\varrho} \circ \kappa_{\tau} \in F_{j}, j=1, \ldots, k, T>\varrho\right) \\
=\frac{1}{u \varrho(x)} \sum_{l=k}^{\infty} \mathbf{P}_{x}\left(\nu^{\emptyset}=l, \gamma_{\zeta-}^{\emptyset} \in d y, \zeta^{\emptyset} \in d s, \zeta^{\emptyset}<\tau^{\emptyset}, \zeta^{\emptyset}<T-\varrho,\right. \\
T \circ \theta_{0}^{j} \circ \kappa_{\tau}<\varrho \text { for } l-k \text { indices } j \in\{1, \ldots, l\}, T \circ \theta_{0}^{j} \circ \kappa_{\tau}>\varrho \\
\left.\quad \text { and } \theta_{0}^{j} \circ c_{\varrho} \circ \kappa_{\tau} \in F_{j} \text { for the } k \text { other indices } j\right) \\
=\frac{1}{u \varrho(x)} \sum_{l=k}^{\infty} \mathbf{P}_{x}^{\mathbf{x}}\left(\gamma_{\zeta-} \in d y, \zeta \in d s, \zeta<\hat{\tau}\right) \mathbf{P}_{x}\left(\nu^{\emptyset}=l \mid \gamma_{\zeta-}^{\emptyset}=y, \zeta^{\emptyset}<\tau^{\emptyset}\right) \\
\quad \times \mathbf{P}_{x}\left(T \circ \kappa_{\tau} \circ \theta_{0}^{j}<\varrho \text { for } l-k \text { indices } j \in\{1, \ldots, l\}, T \circ \kappa_{\tau} \circ \theta_{0}^{j}>\varrho\right. \\
\text { and } c_{\varrho} \circ \kappa_{\tau} \circ \theta_{0}^{j} \in F_{j} \\
\left.\quad \text { for the } k \text { other indices } j \mid \nu^{\emptyset}=l, \gamma_{\zeta-}^{\emptyset}=y, \zeta^{\emptyset}<\tau^{\emptyset}\right)
\end{gathered}
$$




$$
\begin{aligned}
& =\frac{1}{u^{\varrho}(x)} \alpha \hat{\mathbf{P}}_{x}^{\mathbf{x}}\left(\gamma_{s} \in d y\right) d s \sum_{l=k}^{\infty} p_{l}(y)\left(\begin{array}{l}
l \\
k
\end{array}\right)\left(1-u^{\varrho}(y)\right)^{l-k} \\
& \quad \times \prod_{j=1}^{k} \hat{\mathbf{P}}_{y}\left(T \circ \kappa_{\tau}>\varrho, c_{\varrho} \circ \kappa_{\tau} \in F_{j}\right) \\
& =\frac{1}{u^{\varrho}(x)} \alpha \hat{\mathbf{P}}_{x}^{\mathbf{x}}\left(\gamma_{s} \in d y\right) d s \sum_{l=k}^{\infty} p_{l}(y)\left(\begin{array}{l}
l \\
k
\end{array}\right)\left(1-u^{\varrho}(y)\right)^{l-k} u^{\varrho}(y)^{k} \prod_{j=1}^{k} \tilde{\mathbf{P}}_{y}^{\varrho}\left(\omega \in F_{j}\right),
\end{aligned}
$$

where we have used Proposition 1, Markov and branching properties of $X$. Secondly, the case $k=0$ splits in two:

$$
\begin{aligned}
\tilde{\mathbf{P}}_{x}^{\varrho}\left(\nu^{\emptyset}=0, \gamma_{\zeta-}^{\emptyset} \in d y,\right. & \left.\gamma_{\zeta}^{\emptyset}=\Delta, \zeta^{\emptyset} \in d s\right) \\
& =\frac{1}{u^{\varrho}(x)} \mathbf{P}_{x}\left(\nu^{\emptyset}=0, \gamma_{\zeta-}^{\emptyset} \in d y, \zeta^{\emptyset} \in d s, \tau^{\emptyset}>\zeta^{\emptyset}\right) \\
& =\frac{1}{u^{\varrho}(x)} \alpha \hat{\mathbf{P}}_{x}^{\mathbf{x}}\left(\gamma_{s} \in d y\right) d s p_{0}(y)
\end{aligned}
$$

and

$$
\begin{aligned}
\tilde{\mathbf{P}}_{x}^{\varrho}\left(\nu^{\emptyset}=0,\right. & \left.\gamma_{\zeta-}^{\emptyset} \in d y, \gamma_{\zeta}^{\emptyset}=\partial, \zeta^{\emptyset} \in d s\right) \\
& =\frac{1}{u^{\varrho}(x)} \mathbf{P}_{x}\left(\gamma_{s}^{\emptyset} \in d y, \zeta^{\emptyset} \wedge \tau^{\emptyset}>s, \varrho+s \leq T<\varrho+s+d s\right) \\
& =\frac{1}{u^{\varrho}(x)} \hat{\mathbf{P}}_{x}^{\mathbf{x}}\left(\gamma_{s}^{\emptyset} \in d y\right) \mathbf{P}_{y}(\varrho \leq T<\varrho+d s) \\
& =\frac{1}{u^{\varrho}(x)} \alpha \hat{\mathbf{P}}_{x}^{\mathbf{x}}\left(\gamma_{s}^{\emptyset} \in d y\right) d s \frac{a(y, \varrho)}{\alpha} .
\end{aligned}
$$

Combining the above computations with the fact that

$$
\sum_{k=1}^{\infty} \sum_{l=k}^{\infty} p_{l}(y)\left(\begin{array}{l}
l \\
k
\end{array}\right)\left(1-u^{\varrho}(y)\right)^{l-k} u^{\varrho}(y)^{k}=1-F\left(1-u^{\varrho}(y)\right)
$$

gives the desired offspring distribution. Finally, using (3.2), (3.5) and the fact that $v^{u}$ is concentrated on $\mathscr{E}_{\tau}$ it is seen that $\tilde{\mathbf{P}}^{\varrho} \varrho$ governs a branching Markov process of the claimed type. This completes the proof.

4. Assume that the terminal line $\tau$ satisfies the condition (3.4b) above, i.e. $\tau^{\emptyset}=\zeta^{\emptyset}$ a.s. on $\left\{\tau^{\emptyset}<\infty\right\}$. In fact, we are interested in terminal lines generated by variables of the type

$$
\tau^{\emptyset}= \begin{cases}\zeta^{\emptyset}, & \text { if } \nu^{\emptyset} \in I, \gamma_{\zeta-}^{\emptyset} \in A ; \\ +\infty, & \text { otherwise, }\end{cases}
$$

where $I \subseteq\{0,2, \ldots\}, A \in B(E)$. Note that the assumption (A) is not needed for the next result because the non-branching part of $X$ is unaltered when operating with $\kappa_{\tau}$. As above $u^{\varrho}:=\mathbf{P} .(T \geq \varrho)$. 
Theorem 4. Assume that (B)-(E) hold and let $\tau$ be a terminal line of the type (3.16). Then the image of the measure $\hat{\mathbf{P}}$. under the mapping $c_{\varrho}$ is given by $\hat{\mathbf{P}}_{x}^{\varrho}(\omega \in F):=\hat{\mathbf{P}}_{x}\left(c_{\varrho} \circ \omega \in F\right)=\left(1-u^{\varrho}(x)\right) \varepsilon_{\{\dagger\}}(F)+u^{\varrho}(x) \tilde{\mathbf{P}}_{x}^{\varrho}(\omega \in F)$, where $F \in \mathscr{F}_{\infty}$ and the measure $\tilde{\mathbf{P}} \varrho$. governs a branching Markov process having (a) the $u^{\varrho}$-transform of $\hat{\mathbf{x}}$ as the non-branching part,

(b) the offspring distribution: for $k=1,2, \ldots$

$$
\begin{aligned}
\tilde{\mathbf{P}}^{\varrho} .\left(\nu^{\emptyset}=\right. & \left.k \mid \gamma_{\zeta-}^{\emptyset}=y, \gamma_{\zeta}^{\emptyset}=\Delta\right) \\
= & \frac{1}{\mathbf{1}_{A}(y) S_{1}(y)+\mathbf{1}_{A^{c}}(y) S_{2}(y)}\left(\mathbf{1}_{A}(y) \sum_{\substack{l=k \\
l \notin 亠}}^{\infty} p_{l}(y)\left(\begin{array}{l}
l \\
k
\end{array}\right) u^{\varrho}(y)^{k}\left(1-u^{\varrho}(y)\right)^{l-k}\right. \\
& \left.\quad+\mathbf{1}_{A^{c}}(y) \sum_{l=k}^{\infty} p_{l}(y)\left(\begin{array}{l}
l \\
k
\end{array}\right) u^{\varrho}(y)^{k}\left(1-u^{\varrho}(y)\right)^{l-k}\right),
\end{aligned}
$$

for $k=0$

$$
\begin{aligned}
\tilde{\mathbf{P}} \cdot \varrho\left(\nu^{\emptyset}\right. & \left.=0 \mid \gamma_{\zeta-}^{\emptyset}=y, \gamma_{\zeta}^{\emptyset}=\Delta\right)=\frac{p_{0}(y)\left(\mathbf{1}_{I^{c}}(0)+\mathbf{1}_{I}(0) \mathbf{1}_{A^{c}}(y)\right)}{\mathbf{1}_{A}(y) S_{1}(y)+\mathbf{1}_{A^{c}}(y) S_{2}(y)}, \\
\tilde{\mathbf{P}} \cdot \varrho\left(\gamma_{\zeta}^{\emptyset}\right. & \left.=\Delta \mid \gamma_{\zeta-}^{\emptyset}=y\right)=1-\tilde{\mathbf{P}} \varrho .\left(\gamma_{\zeta}^{\emptyset}=\partial \mid \gamma_{\zeta-}^{\emptyset}=y\right) \\
& =\frac{\mathbf{1}_{A}(y) S_{1}(y)+\mathbf{1}_{A^{c}}(y) S_{2}(y)}{(a(y, \varrho) / \alpha)+\mathbf{1}_{A}(y) S_{1}(y)+\mathbf{1}_{A^{c}}(y) S_{2}(y)}
\end{aligned}
$$

where 1. denotes the indicator function of a set and

$$
\begin{aligned}
& S_{1}(y)=\sum_{\substack{l=0 \\
l \notin I}} p_{l}(y)-\sum_{\substack{l=1 \\
l \notin I}} p_{l}(y)\left(1-u^{\varrho}(y)\right)^{l}, \\
& S_{2}(y)=p_{0}(y)+1-F\left(1-u^{\varrho}(y)\right) .
\end{aligned}
$$

Proof being very similar to the proof of Theorem 3 , is omitted. However, we point out, using the notations in (3.12), that

$$
\begin{aligned}
T_{2}:= & \mathbf{P}_{x}\left(T<\varrho, \zeta^{\emptyset}<t, \tau^{\emptyset}<t\right)+\mathbf{P}_{x}\left(T<\varrho, \zeta^{\emptyset}<t, \tau^{\emptyset}>t\right) \\
= & \mathbf{P}_{x}\left(T<\varrho, \zeta^{\emptyset}<t, \tau^{\emptyset}=\zeta^{\emptyset}\right)+\mathbf{P}_{x}\left(T<\varrho, \zeta^{\emptyset}<t, \tau^{\emptyset}=\infty\right) \\
= & \mathbf{P}_{x}\left(\nu^{\emptyset} \in I, \gamma_{\zeta-} \in A, \zeta^{\emptyset}<t\right) \\
& \quad+\int_{0}^{t} \int_{A^{c}} \mathbf{P}_{x}^{\mathbf{x}}\left(\zeta \in d s, \gamma_{\zeta-} \in d y\right) \sum_{l=2}^{\infty} p_{l}(y)\left(\mathbf{P}_{y}(T<\varrho-s)\right)^{l} \\
& \quad+\int_{0}^{t} \int_{A} \mathbf{P}_{x}^{\mathbf{x}}\left(\zeta \in d s, \gamma_{\zeta-} \in d y\right) \sum_{\substack{l=2 \\
l \notin I}} p_{l}(y)\left(\mathbf{P}_{y}(T<\varrho-s)\right)^{l},
\end{aligned}
$$

and this gives, as in the proof of Proposition 2, the desired exit distribution. 
Remark. When operating with $c_{\infty}$ the results above remain valid. In this case, of course, the assumption (E) is not needed and the function $a$ vanishes in the statements.

\section{References}

[1] Asmussen, S., and H. Hering: Branching processes. - Birkhäuser, Boston-Basel-Stuttgart, 1983.

[2] Athreya, K.B., and P.E. NeY: Branching processes. - Springer-Verlag, Berlin-Heidelberg-New York, 1972.

[3] Chauvin, B.: Product martingales and stopping lines for branching Brownian motion. Preprint no. 26, Université Pierre et Marie Curie, Laboratoire de Probabilités.

[4] Dynkin, E.B.: The space of exits of a Markov process. - Russian Math. Surveys 24:4, 1969, 89-157 (English translation of Uspekhi Mat. Nauk 24:4, 1969, 89-152).

[5] HARRIS, T.E.: Branching processes. - Ann. Math. Statist. 19, 1948, 474-494.

[6] Hering, H.: The non-degenerate limit for supercritical branching diffusions. - Duke Math. J. $45: 3,1978,561-600$.

[7] Ikeda, N., M. Nagasawa, and S. Watanabe: Branching Markov processes III. - J. Math. Kyoto Univ. 9:1, 1969, 95-160.

[8] Neveu J.: Arbes et processus de Galton-Watson. - Ann. Inst. H. Poincaré Probab. Statist. 22:2, 1986, 199-207.

[9] Neveu, J.: Erasing a branching tree. - Adv. in Appl. Probab. 1986, suppl. 101-108.

[10] Salminen, P.: A ratio limit theorem for erased branching Brownian motion. - Stochastic Process. Appl. (to appear).

[11] Sharpe, M.: General theory of Markov processes. - Academic Press, Inc., San Diego, 1988.

[12] Williams, D.: Diffusions, Markov processes and martingales. - Wiley and Sons, London, 1979. 